Revue musicale OICRM

revue musicale oicrm

\title{
Participer. Essai sur les formes démocratiques de la participation, de Joëlle Zask
}

\section{Marc-Antoine Boutin}

Volume 7, numéro 2, 2020

URI : https://id.erudit.org/iderudit/1072422ar

DOI : https://doi.org/10.7202/1072422ar

Aller au sommaire du numéro

Éditeur(s)

Observatoire interdisciplinaire de création et recherche en musique (OICRM)

\section{ISSN}

2368-7061 (numérique)

Découvrir la revue

Citer ce compte rendu

Boutin, M.-A. (2020). Compte rendu de [Participer. Essai sur les formes démocratiques de la participation, de Joëlle Zask]. Revue musicale OICRM, 7(2), 143-149. https://doi.org/10.7202/1072422ar d'utilisation que vous pouvez consulter en ligne.

https://apropos.erudit.org/fr/usagers/politique-dutilisation/ 


\title{
Participer. Essai sur les formes démocratiques de la participation, de Joëlle Zask Lormont, Le Bord de l'eau, 2011, 326 pages
}

\author{
Marc-Antoine Boutin
}

Mots clés : art et démocratie ; compte rendu ; médiation culturelle ; philosophie politique ; Joëlle Zask.

Keywords: art and democracy; cultural mediation; political philosophy; review; Joëlle Zask.

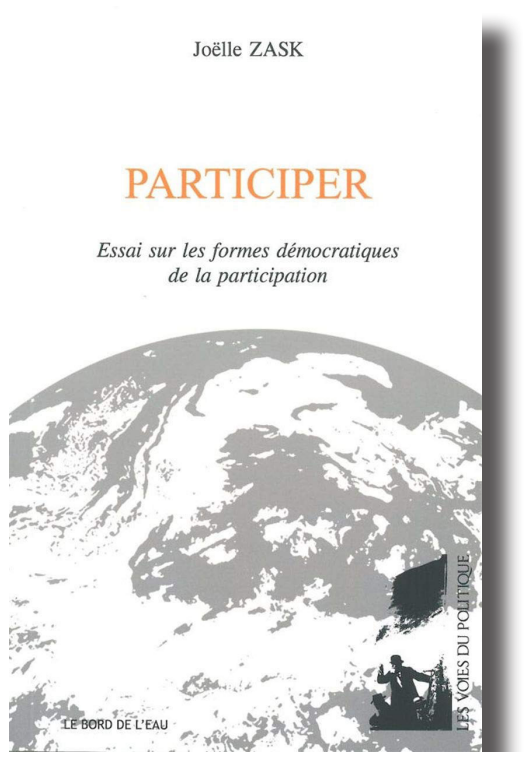
En art, en politique, à l'école, dans l'entreprise, dans la presse ou sur le web, une même injonction est aujourd'hui adressée à chacun d'entre nous : participez ! Oui, mais à quoi, comment et dans quel but?
Que signifie, que sous-entend, qu'engage la participation ? Sait-on ce que participer veut dire $^{1}$ ?

Telles sont les questions que pose d'entrée de jeu Joëlle Zask, spécialiste de philosophie politique et de philosophie pragmatiste, dont celle de John Dewey, professeure à l'Université d'Aix-Marseille et auteure de Art et démocratie. Les peuples de l'art ${ }^{2}$, Outdoor Art. La sculpture et ses lieux ${ }^{3}$ et Quand la place devient publique ${ }^{4}$.

1 Joëlle Zask (2011), Participer. Essai sur les formes démocratiques de la participation, Lormont, Le Bord de l'eau, quatrième de couverture.

2 Joëlle Zask (2003), Art et démocratie. Les peuples de l'art, Paris, Presses Universitaires de France.

3 Joëlle Zask (2013), Outdoor Art. La sculpture et ses lieux, Paris, La Découverte.

4 Joëlle Zask (2018), Quand la place devient publique, Lormont, Le Bord de l'eau. 
La recension de cet ouvrage ${ }^{5}$ traitant de la participation trouve naturellement sa place dans ce numéro de la Revue musicale OICRM dédié à la médiation de la musique, car Zask y déploie la réponse à des questions qui se posent avec force dans ce domaine, voire qui lui sont fondamentales. Si les réflexions dans Participer sont surtout adaptées au contexte politique, cette recension montrera donc qu'elles sont transposables à tous les domaines (comme le suggère la citation en exergue du texte) et plus particulièrement à celui de la médiation culturelle.

L'essai s'articule autour de deux axes de force qui permettent de mieux comprendre les conditions d'existence d'une "réelle participation " et d'ainsi préciser le sens de ce vocable parfois galvaudé. Dans le contexte actuel où la participation au politique est couramment confondue avec un " mécanisme dont le but est de tirer le meilleur parti possible des gens au profit d'une entreprise dont les finalités ne sont pas de leur ressort " (p. 8), l'auteure rappelle en premier lieu que, dans une démocratie libérale, les individus doivent pouvoir, par définition, " exercer réellement une influence sur les conditions qui les affectent " (p. 9). Il est d'autant plus problématique de "faire croire à la participation » (p. 9), souligne-t-elle. Dans un mouvement de revalorisation de la participation, Zask s'attache, en second lieu, à mettre en évidence ce qui la caractérise réellement : la réciprocité entre l'individuel et le collectif. Cette réciprocité est le seul moyen pour s'accomplir soi-même et se perfectionner en tant que citoyen ; en somme, le seul moyen pour les individus de réaliser leur individualité (p. 237).

L'ouvrage est structuré en fonction des trois modes - ou trois " types d'expériences »-complémentaires et constitutifs d'une vraie participation : " prendre part », " apporter une part » (contribuer) et " recevoir une part» (bénéficier). Si une participation idéale se traduit par la réciprocité entre ces trois compléments, elles sont en réalité trop souvent séparées - cette désunion étant «la source de toutes les injustices » (p. 14). Zask se réfère à de nombreux philosophes de la démocratie, tels John Dewey, Alexis de Tocqueville, Thomas Jefferson et Aristote, mais aussi à des anthropologues, sociologues, psychanalystes et économistes tels Edward Sapir, Bronisław Malinowski, Marcel Mauss, Karl Marx, Donald Winnicott et Amartya Sen.

Dans la première partie, "Prendre part » (p. 15-114), Zask nous invite à réfléchir aux caractéristiques principales du " prendre part », première condition à une réelle participation. Dans un premier temps, l'auteure distingue le « prendre part » $d u$ « faire partie». Partant de l'analyse d'évènements historiques et politiques, l'auteure démontre que le premier, "prendre part ", se caractérise par l'organisation d'une activité en commun dont le fait d'être ensemble « est le fruit d'une démarche personnelle et en partie volontaire » (p. 17), alors que l'autre, "faire partie ", efface l'individualité des membres au profit de l'entité. Pour illustrer cette différence, Zask donne tout d'abord l'exemple de la "Commune » comme étant l'association politique dans laquelle le "prendre part [...] est assuré au plus haut point » (p. 77) et celui de l'autogouvernement comme le moyen d'assurer l'autonomie et la liberté des citoyens. A contrario, le cas de la «fraternité » permet d'illustrer une situation où « les droits et les devoirs 
individuels et institués [ne se fondent pas] sur une exigence de réciprocité » (p. 65), ni d'égalité, mais reposent sur le respect d'une filiation reposant sur une allégeance inconditionnelle. En somme, le fait de " prendre part " à une entreprise sociale met en évidence la nature particulière des liens unissant les membres d'un groupe et repose sur des conditions particulières permettant la «communalisation » (p. 61) : la création du commun est " la raison d'être du groupe » (p. 151). Il faut ici souligner toute l'importance qu'a ce postulat pour la médiation de la musique : la musique ne constitue pas le commun en " soi », mais elle le devient à travers la participation des membres du groupe. Mais alors comment cette musique devient-elle un "commun " en situation de participation ? En autres termes, comment se traduit ce processus de « communalisation »? On trouvera réponse à cette question avec la deuxième modalité de la participation, "apporter une part".

Dans la deuxième partie de l'ouvrage, «Contribuer (apporter une part)» (p. 115-218), la focale est moins sur le groupe que sur l'individu : la contribution se définit comme un « acte d'investissement personnel» (p. 151) et correspond à l'engagement de l'individu au commun. En effet, la valeur essentielle de la contribution est de " marquer, même à un degré modeste, les relations sociales » (p. 127). Cette " marque » (Zask emploie «trace ») est laissée lorsque nous nous emparons d'une opportunité mise à disposition dans une situation de participation pour « influencer les décisions collectives qui nous concernent » (p. 286). Ainsi, " prendre part » et " apporter une part » se distinguent $\mathrm{du}$ fait que le premier a la fonction première « de maintenir des structures sociales stables sans être toutefois rigides", tandis que le second a la fonction de "provoquer $\mathrm{du}$ "changement social" »(p. 118). Comme contre-exemple à la contribution, Zask mobilise le concept de « superfluité ». Une personne «superflue » est une personne

dont l'action n'est jamais qu'une agitation, parce qu'elle n'aboutit à rien [ou] ne s'incarne dans aucune situation concrète [...]. L'importance de la contribution peut donc être repérée a contrario à travers les situations dont précisément toute trace de l'action individuelle a disparu, qu'elle est été trop ténue, qu'elle n'ait compté pour personne ou qu'elle se soit effacée (p. 155).

Chez Marx $^{6}$, par exemple, la superfluité résulte de causes socioéconomiques et " s'installe dès que les possibilités de contribution individuelle sont supprimées » (p. 158).

Dans la troisième et dernière partie (p. 219-320), il est question des " bénéfices », soit du fait de « recevoir une part » du groupe. Au diapason avec les deux premières conditions de la participation, le bénéfice « est tel s'il favorise l'individuation ${ }^{7}$ de son

6 L'auteure se réfère aux Manuscrits de 1844 de Karl Marx (1844), édition électronique réalisée à partir de l'édition d'Émile Bottigelli (présentation, traduction et notes) (1972), Paris, Les Éditions sociales, http://classiques.uqac.ca/classiques/Marx karl/manuscrits 1844/Manuscrits 1844.pdf, consulté le 11 septembre 2019.

7 "L'individuation, pour Zask, n'est pas un "bien" qui puisse être possédé mais une qualité de l'existence humaine» (p. 221). Si elle est un bénéfice et une finalité en soi, l'individuation ne peut pas être atteinte ou accomplie complètement, mais au contraire peut être approfondie et perfectionnée. Il faut plutôt concevoir l'individuation comme un « continuum d'expériences » (p. 222). 
récipiendaire - si, par conséquent, il procure à l'individu les moyens lui permettant de prendre part et de contribuer » (p. 226). C'est là que se trame le processus d'interinfluence entre les individus qu'est la réciprocité sociale : le " pouvoir de contribuer ne dépend pas de qualités strictement individuelles, mais d'une forme d'intégration entre de l'individuel et du social » (p. 134). En autres mots, les composantes de la participation sont nécessairement complémentaires : pour pouvoir contribuer, il faut bénéficier (en tant que forme de bénéfice, la reconnaissance est un bon exemple), de même que pour pouvoir bénéficier, il faut contribuer.

Il mérite de souligner l'emploi du terme «intégration » sociale par l'auteure, puisque dans les recherches et la pratique de la médiation culturelle le terme "inclusion » est généralement préféré. Une démarche inclusive consiste à mobiliser des «logiques de pluralisation des voix ${ }^{8}$ » et permet ainsi de «faire valoir sa personnalité, ses talents et ses idées pour contribuer au projet de société ${ }^{9}$ ". Alors qu'en situation d'intégration ${ }^{10}$, les individus sont en quelque sorte " exclu·e.s de l'intérieur ${ }^{11}$ ", l'inclusion "repose sur une représentation "dénormalisée" de la diversité (AuCoin et Vienneau, 2010)" ${ }^{12}$. Selon la définition que donne Zask de " prendre part ", le terme d' " inclusion » nous semble plus juste que celui d' "intégration ».

Pour montrer qu'une participation idéale se déroule dans un environnement " socioculturel donné » (p. 228), Zask se réfère aux travaux de Donald Winnicott et au concept d'environnement «suffisamment bon ». Dans de tels environnements, les bénéfices sont pour les individus "plus que des chances ou des possibilités », ce sont des « opportunités» (p. 241). Chez l'économiste Amartya Sen, l'environnement doit mettre à disposition des " capabilités ", soit des " possibilités concrètes d'usages ${ }^{13}$ ».

8 Voir Iris Marion Young (2000), Inclusion and Democracy, New York, Oxford University Press.

9 Voir Regroupement québécois de la danse (2019), Comprendre les enjeux de l'inclusion en danse. Lexique commenté, p. 8, https://www.quebecdanse.org/images/upload/files/lexique inclusion rqd final web 2019.pdf, consulté le 4 décembre 2019.

10 Le terme d' " intégration » sociale, en sociologie, repose sur une conception duale du monde social : « le système et l'individu (ou encore la société et les individus), qui engagent les individus à adopter subjectivement les comportements moralement requis par les institutions ». Sylvia Faure (2014), " Médiation, intégration, socialisation : que veulent dire les mots ? ", dans Frédrique Montandon et Thérèse Pérez-Roux (dir.), Les médiations culturelles et artistiques. Quels processus d'intégration et de socialisation?, Paris, L'Harmattan, p. 19-26.

11 Voir Pierre Bourdieu et Patrick Champagne (1992), "Les exclus de l'intérieur ", Actes de la recherche en sciences sociales, vol. 91-92, p. 71-75, https://www.persee.fr/doc/arss 0335-5322 1992 num 9113008 , consulté le 4 décembre 2019.

12 Nadia Rousseau et al. (2015), Les enjeux de l'intégration et de l'inclusion scolaire des élèves à risque du primaire et du secondaire : méta-analyse et méta-synthèse, Rapport de recherche, Programme " Actions concertées ", Fonds de recherche du Québec - Société et Culture, p. 4, http://www.frqsc.gouv.qc.ca/ documents/11326/448958/PC RousseauN rapport integration-inclusion.pdf/65f4f932-3595-448a-a8d8db22b1df32b9, consulté le 4 décembre 2019.

13 "Offrir un livre à un aveugle n'est pas adapté à ce que le grand économiste Amartya Sen a appelé ses "capabilités" (qui sont, contrairement aux capacités, des possibilités concrètes d'usages dans un environnement particulier) », dans Joëlle Zask (2018), Participer, qu'est-ce que ça veut dire?, site Internet de la société française de prospective, https://www.societefrancaisedeprospective.fr/joelle-zask-participer-quest-ce-quecela-veut-dire/, consulté le 8 septembre 2019. 
Ces notions sont directement liées à celle d'encapacitation (empowerment) que la médiation culturelle met en son cœur. L'étymologie même du terme " médiation » culturelle renvoie à cette notion d'encapacitation qui reconnaît l'originalité de chaque voix - concordant, de fait, avec les fondements d'une démarche inclusive. Comme le dit Sylvie Pébrier, inspectrice générale au ministère de la Culture (France),

la force du mot médiation est sa racine « média », c'est-à-dire " entre ». C'est un mot qui permet de bien penser la mise en vis-à-vis d'un artiste, [d'un objet culturel] et d'une personne, [laquelle doit être entendue] non pas comme un spectateur passif ou un réceptacle sans culture, mais quelqu'un qui arrive aussi avec sa propre identité culturelle et donc de ne plus penser qu'il y aurait un transfert de l'un vers l'autre, comme souvent dans les logiques d'accès, mais bien quelque chose qui va naître de cette rencontre, qui est inédit ${ }^{14}$.

En conclusion, c'est au nom de la liberté et de la justice - le droit de chacun à jouir des conditions nécessaires à son individuation - que Zask pose en priorité, voire un "devoir de justice » (p. 285), le fait de veiller à la combinaison et à l'équilibre des formes de la participation. Autrement dit, il faut s'attarder à la " production sociale des conditions de l'individuation » (p. 323). C'est là tout l'intérêt de Participer, et ce qui lui attribue son originalité, que de se pencher sur les " conditions d'une réelle participation ». En ce sens, il peut se lire comme un "guide éthique » de la participation pour les médiateurs et médiatrices qui sont parfois mal outillé·e·s. Si d'autres types de questions se poseraient alors - par exemple, comment évaluer si telle action individuelle a suffisamment marqué le commun? Sur la base de quels critères juger de la qualité d'une reconnaissance ? - elles seraient d'un autre ordre, consistant davantage en la catégorisation des " niveaux de participation ${ }^{15}$ ».

En effet, 1'hypothèse est que lorsqu'on comprend ce que signifie réellement participer, " on dispose d'un critère à la fois général et concret pour décider d'une politique, pour évaluer la légitimité des normes et des institutions de notre société, ou encore pour sonder la justesse de nos convictions morales » (p. 323). Dans le domaine culturel, par exemple, une telle conception de la participation pourrait permettre l'édification d'une politique plus démocratique de la culture ${ }^{16}$, c'est-à-dire une politique qui n'est pas destinée à nommer et favoriser une culture particulière, mais qui s'assure de mettre en place un environnement culturel favorable à une diversité de pratiques, lesquelles tiendront du ressort des citoyens pour éclore :

Le but d'une politique culturelle n'est pas de distribuer les mêmes produits culturels à tout le monde, ou d'amener le maximum de gens vers tel secteur de consommation

14 Conseil économique social et environnemental (2017), "Questions à Sylvie Pébrier (Ministère culture et communication) ", YouTube, https://www.youtube.com/watch?v=MxJfeJBlTtA, consulté le 4 décembre 2019.

15 Nathalie Casemajor, Ève Lamoureux et Danièle Racine (2016), " Art participatif et médiation culturelle. typologie et enjeux des pratiques ", dans Cécile Camart et al. (dir.), Les mondes de la médiation culturelle, «Vol. 1. Approches de la médiation », Paris, L'Harmattan, p. 171-184.

16 Joëlle Zask (2016), « De la démocratisation à la démocratie culturelle», Nectart, vol. 3, n 2, p. 44. 
culturelle pour justifier les dépenses publiques qui lui ont été consenties [...]. [Au contraire, son but est] d'influer sur les institutions, les habitudes, les logiques privées ou publiques qui tendent soit à confisquer les ressources d'individuation, soit à les supprimer [et] d'infléchir la vie culturelle afin que soient redistribuées à tous, et le plus également possible, les ressources d'individuation dont chacun a besoin pour que sa vie ait un sens ${ }^{17}$.

Nous avons encore trop tendance à considérer la puissance publique d'un point de vue " utilitariste ${ }^{18}$ ", écrit Zask, lui assignant le mandat " de nous procurer des choses qui nous importent et qui comblent nos désirs tels qu'ils se sont manifestés indépendamment de nos associations » (p. 280). Or, ajoute-t-elle, il faut comprendre que « la citoyenneté [est] une affaire d'éducation et de culture dont la transmission dépend de la communauté organisée et dont l'actualisation dépend de la participation active des individus aux expériences qu'ils partagent les uns avec les autres » (p. 204). Le fait de participer et surtout de pouvoir participer n'est donc pas accessoire, mais est au contraire essentiel.

En observant l'action culturelle de plus près, augmentant la proximité avec les publics, on se rapproche des initiatives de médiation culturelle. Ces instances ont-elles aussi le pouvoir - et le devoir, pourrait-on dire avec Zask - de créer des opportunités d'échange et de participation entre les publics et les objets culturels ? Ces réflexions éclairent ce que l'on pourrait nommer une "responsabilité » qu'ont les professionnel·le's de la culture. En faisant le lien avec le domaine musical, on pose alors : comment permettre aux participant·e·s d'ateliers de médiation de la musique de " prendre part ", " apporter une part » et « recevoir une part »? En effet, si participer c'est " avoir un mot à dire concernant la vie commune et l'opportunité, en termes d'écoute, de lieu, de compétence [...], de le dire effectivement, et de manière à ce qu'il soit entendu ${ }^{19}$ ", avec Bruno Péquignot, on peut affirmer que les médiateurs culturels sont, de par leur fonction, les défenseurs de cette conviction qui accorde une priorité à l'individuation des publics :

17 Joëlle Zask (2009), «Propositions pour une politique culturelle», Cahiers Sens public, vol. 11-12, n 3, p. 114-115.

18 Dans son objectif de définition du «bénéfice », Zask développe une « Comparaison entre la participation et le "don" » (p. 221-235). L'auteure rappelle que dans son Essai sur le don (1925), Marcel Mauss « aborde le don comme le système économique et juridique le plus ancien » (p. 229). Ce système est caractérisé par l' "échange-don ", une "triple obligation » consistant à donner, prendre et rendre, et correspond " dans une certaine mesure » à prendre part, bénéficier et contribuer (p. 230). Ces prestations et contre-prestations « ne sont ni intéressées, n'étant justifiées par la recherche rationnelle de l'utilité individuelle, ni désintéressées, étant destinées à consolider les relations sociales » (p. 233). Cela fait directement écho au dernier ouvrage d'Alain Caillé, sociologue et directeur de La Revue du M.A.U.S.S. (Mouvement anti-utilitariste en sciences sociales), intitulé Extensions du domaine du don. Demander-donner-recevoir-rendre (Arles, Actes Sud, 2019). À travers une relecture des travaux de Mauss sur le "don ", Caillé réactualise cette " triple obligation » et argumente qu'elle « régit en réalité l'essentiel de nos rapports aux autres » (quatrième de couverture du livre).

19 Joëlle Zask (2007), «Art et démocratie sont-ils antinomiques? », L'Observatoire, vol. 41, n 2, p. 53. 
La fonction des médiateurs culturels est donc bien cadrée, ils agissent au sein des institutions pour provoquer une mise en cause des évidences idéologiques [...] qui, imposant un mode du voir, du lire, de l'écouter, nous rendent aveugles, illettrés et sourds à la nouveauté proposée par l'artiste. [Cette fonction] n'est pas de traduire en langage commun ce que l'artiste [avec son art] s'est acharné à dire autrement, mais [d'amener] le public à interroger cet autrement ${ }^{20}$.

En définitive, la " démocratisation ${ }^{21}$ » de la culture pour Zask devrait revenir à " répartir le plus égalitairement possible les ressources d'individuation ». Ainsi, les actions de médiation culturelle engageant une participation ne sont donc pas normatives, mais favorisent un environnement "suffisamment bon ", ouvert et accessible aux réflexions critiques, où chacun peut " contribuer » de par ses propres compétences et «bénéficier» de l'interaction avec autrui. Les notions de commun, de droits culturels et de sociabilité, qui sont au cœur du propos de l'auteure, sont aussi à considérer dans les réflexions en médiation culturelle ${ }^{22}$.

20 Bruno Péquignot (2007), « Sociologie et médiation culturelle », L'Observatoire, vol. 32, n 2, p. 6-7.

21 Zask est critique du terme " démocratiser », dont la connotation est aujourd'hui très forte, et précise que cela ne signifie « ni vouloir éduquer les masses prétendument incultes en mettant à portée les chefsd'œuvre de l'élite, ni chercher à leur plaire pour s'assurer de leur adhésion, consommatrice ou électorale ». Pour l'auteure, on peut conserver l'expression " démocratiser la culture » à condition que cela signifie une répartition égalitaire des ressources d'individuation, dans Eric Demey (propos recueillis par) (2017), « Pour une nouvelle définition de la culture », entretien avec Joëlle Zask, La Terrasse, no 256 (27 juin), https:// www.journal-laterrasse.fr/pour-une-nouvelle-definition-de-la-culture/, consulté le 11 septembre 2019.

22 À ce sujet, on peut notamment consulter : Frédérique Montandon et Thérèse Pérez-Roux (dir.) (2014), Les médiations culturelles et artistiques. Quels processus d'intégration et de socialisation?, Paris, L'Harmattan. 